\title{
On the Use of Time-Frequency Reassignment and SVM-Based Classifier for Audio Surveillance Applications
}

\author{
Souli S. Sameh \\ Signal, Image and pattern recognition research unit, ENIT/ \\ Dept. of Genie Electrique \\ BP 37, 1002, Le Belvédère, Tunisia \\ soulisameh@yahoo.fr \\ Lachiri Z. Zied \\ Dept. of Physique and Instrumentation, INSAT/ \\ BP 676, 1080, Centre Urbain, Tunisia \\ ziedlachiri@enit.rnu.tn
}

\begin{abstract}
In this paper, we propose a robust environmental sound spectrogram classification approach. Its purpose is surveillance and security applications based on the reassignment method and log-Gabor filters.

Besides, the reassignment method is applied to the spectrogram to improve the readability of the timefrequency representation, and to assure a better localization of the signal components. Our approach includes three methods. In the first two methods, the reassigned spectrograms are passed through appropriate log-Gabor filter banks and the outputs are averaged and underwent an optimal feature selection procedure based on a mutual information criterion. The third method uses the same steps but applied only to three patches extracted from each reassigned spectrogram. The proposed approach is tested on a large database consists of 1000 sounds belonging to ten classes. The recognition is based on Multiclass Support Vector Machines.
\end{abstract}

Index Terms-Environmental sounds, Reassignment method, Gabor filters, SVM Multiclass, Mutual Information.

\section{INTRODUCTION}

Generally, automatic sound recognition (ASR) has been interested on the analysis of speech and music but now some efforts have been emerged on detecting and classifying environmental sounds [1-2-3-4] .This paper is interested in the environmental sounds classification. The purpose is the identification of some current life sounds class. Among the possible applications [5-6-7-8], we quote: the cars classification according to their noise, the fire arms sounds identification to warn the police, the distress sounds identification for the remote monitoring systems and medical security [9]. Many previous works on environmental sounds classification have proposed a variety of acoustic features such as MFCCs, frequency roll-off, spectral centroid, zero-crossing, energy, LinearFrequencies Cepstral Coefficients (LFCCs), parameters from wavelet transform. These descriptors can be used as a combination of some, or even all, of these 1-D audio features together, but sometimes the combination between descriptors increases the classification performance compared with the individually-used features. This increase is explained by the presence of many features which negatively influenced the quality of classification. Therefore, the recognition rate can be decreased when the number of targeted classes increases because the presence of some difficulties likes randomness and high variance [1]. Recently, some efforts have emerged in the new research direction, which demonstrate that the visual techniques inspired from processing image can be applied in musical sounds [1011], and show that the descriptors can be extracted from the spectrogram instead of extracting from the signal [1213]. In order to explore the visual information of environmental sounds, our own previous work [14], consist in integrating the audio texture concept as image texture, the feature extraction method uses the structure time-frequency by means of translation-invariant wavelet decomposition and a patch transform alternated with two operations: local maximum and global maximum to reach scale and translation invariance. The aim is to find efficient parameters. The use of the time-frequency representation in signal processing domain becomes very large and wide. It is explained by the fact of presence of a large amount information and the easily interpretation of this representation [12]. However, the spectrogram has some disadvantage, the windowing operation required in spectrogram computation introduces an unsavory tradeoff between time and frequency resolution, so spectrogram provides a time-frequency representation that is blurred in time, in frequency, or in both dimensions [15].

Hence, the reassignment method intervenes to 
resolve problems caused by the use of spectrogram. The spectrogram reassignment is an approach for refocusing the spectrogram by mapping the data to time-frequency coordinates that are nearer to the true region of the analyzed signal support [15]. In addition, the reassignment method and its application fields for detecting and classifying non stationary signal is now well determined, and wide-spread [16]. This paper is interested in log-Gabor filters application to the reassigned spectrogram. Besides, many studies like [1718] show that spectro-temporal modulations play an important role in sound perception and stress recognition in speech [19], in particular the 2D Gabor, which is suitable and very efficient to feature extraction.

In the recognition patterns, especially in image classification, Gabor filters offer an excellent simultaneous localization of spatial and frequency information [20]. They have many useful and important properties, in particular the capacity to decompose an image into its underlying dominant spectro-temporal components. The Gabor filters represent the most effective means of packing the information space with a minimum of spread and hence a minimum of overlap between neighboring units in both space and frequency [13]. We developed here, three methods based on spectro-temporal components. The First method begins with a reassigned spectrogram calculation, which then goes through a single log-Gabor filter, and finally passed goes through an optimal feature procedure based on mutual information. The second method is similar to the first one but in this case, with an averaged 12 log-Gabor filters. In the third method, we divide the reassigned spectrogram into 3 patches, and then we apply the second method for each spectrogram. During the classification phase, we use the SVM's with multiclass approach: OneAgainst-One.

Section 2 present a description of the adopted approach in the context of the reassigned spectrogram and logGabor filters followed then by a brief history of reassignment method and log-Gabor filters approach Classification results are reported in Section 3 to demonstrate the performance of our approach and finally conclusions are presented in Section 4.

\section{ENVIRONMENTAL SOUND CLASSIFICATION SYSTEM BASED ON REASSIGNMENT METHOD AND LOG-GABOR FILTERS}

Our environmental sound classification system is composed of three methods. In the first method, a reassigned spectrogram is generated from sound. Next, it goes through single log-Gabor filter extraction for 2 scales $(1,2)$ and six orientations $(1,2,3,4,5,6)$ that corresponding consecutively

$\left(0^{\circ}, 18^{\circ}, 36^{\circ}, 54^{\circ}, 72^{\circ}, 90^{\circ}\right)$. Then, we apply mutual information in order to get an optimal feature. This feature is finally used in the classification.

The second method consists of the same steps as first one, but with an averaged $12 \log$-Gabor filters instead of single log-Gabor filter.

In the third method the idea is to segment each reassigned spectrogram into 3 patches. Intuitively, for each reassigned spectrogram patch, averaged 12 logGabor filters are calculated. After that we apply a mutual information selection to pass finally in the classifier. In the classification phase, we use SVM, in One-AgainstOne configuration with the Gaussian kernel.

\section{A. Features Extraction Methods}

The majority of studies utilize audio descriptors for classification system, especially the popular MFCC, but some studies like [11-19-21-22] have choose features based on a technique mainly used in images processing, the use of visual features give an excellent result for classification for speech, and musical sounds, this result motivated us to test these descriptors on the environmental sounds. The feature extraction is based on three methods.

\section{- Single log-Gabor filter applied to reassigned spectrogram}

The procedure for generating the single log-Gabor filter is shown in "Fig.1".This approach consists in computing 12 log-Gabor filters that are derived from the environmental sounds reassigned spectrograms, with 2 scales $(1,2)$ and 6 orientations $(1,2,3,4,5,6)$, this extraction allows the best correlation of signal structures. Then, for each single filter result we calculated the magnitude, after that, we use mutual information (MI) algorithm to find an optimal feature vector that is later used during the classification phase [20].

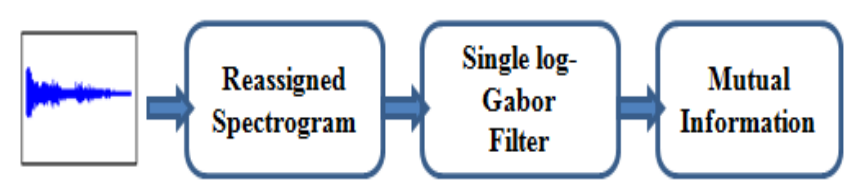

Fig.1. Feature extraction using single log-Gabor filter

\section{- 12 log-Gabor Filters concatenation applied to reassigned spectrogram}

In this method, we tested log-Gabor filters for different concatenation numbers of log-Gabor filters $N_{f}=$ $2,3,4, \ldots, 20$ the best result is obtained for concatenation of 12 first log-Gabor filters that is for $N_{f}=12$.

In this method, each environmental sound reassigned spectrogram goes through a bank of 12 log-Gabor filters. This produces a bank of 12 log-Gabor filters $\left\{G_{11}, G_{12}, \ldots, G_{16}, G_{21}, \ldots, G_{25}, G_{26}\right\}$, with each filter representing different scales and orientations. Thus, this result allows us to say that we obtain for each spectrogram a bank of 12 log-Gabor filters. These resulting feature values are later concatenated into 1Dvectors. Then the average computation is analysed according to the MI criteria, and is sent to SVM for classification ("Fig.2"). 


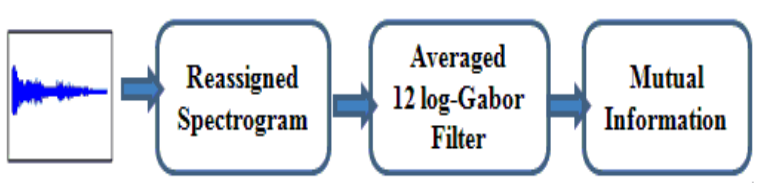

Fig.2. Feature extraction using 12 log- Gabor filters

\section{- Three Reassigned Spectrogram Patches with 12 log-Gabor Filters}

The method consists in using the reassigned spectrogram patch. The aim is to find the suitable part of spectrogram, where the efficient structure concentrates, which gives a better result. We tested our method using log-Gabor filter for three spectrogram patches. We tested for patch number $N_{p}=2,3,4,5$, we remark that the satisfactory result is obtained for $N_{p}=3$. The idea is to extract three patches from each reassigned spectrogram. The first patch included frequencies from $0.01 \mathrm{~Hz}$ to $128 \mathrm{~Hz}$, the second patch, from $128 \mathrm{~Hz}$ to $256 \mathrm{~Hz}$, and the third patch, from $256 \mathrm{~Hz}$ to $512 \mathrm{~Hz}$. Indeed, each patch goes through 12 log-Gabor filters, followed by an average operation and then, MI feature selection algorithm is used, which constitutes the parameter vector for the classification ("Fig.3").

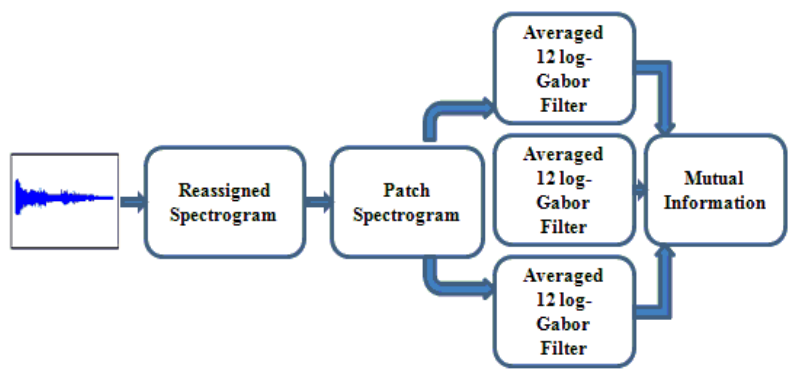

Fig.3. Feature extraction using 3spectrogram patches with 12 log-Gabor filters

\section{B. The Reassignment Method}

The reassignment idea was presented first by Kodera et al. [3], then widens to the Cohen class of bilinear timefrequency energy distributions and to the refine class by Auger et al. and the affine smoothed pseudo Wigner-Ville distribution [17 16].

Besides, reassignment method allows the moving of the spectrogram values away from their computation place. In addition, it focuses energy components by moving each time-frequency location to its group delay and instantaneous frequency [23].

The spectrogram is the square modulus of the Short Time Fourier Transform $\operatorname{STFT}_{h}(x ; t, \omega)$

$$
\begin{gathered}
S_{h}(x ; t, \omega)=\left|\operatorname{STFT}_{h}(x ; t, \omega)\right|^{2} \\
\operatorname{STFT}_{h}(x ; t, \omega)=\int_{-\infty}^{+\infty} x(u) h^{*}(t-u) e^{-j \omega u} d u
\end{gathered}
$$

Moreover, the Time-frequency representation is more suitable and efficient for non-stationary signals.

Nevertheless, this representation has certain disadvantages. This disadvantage is manifested by its unseparable kernel allowing the spreads of the time and frequency smoothings bound, and even opposed [16], which leads to the spectrogram a loss of resolution and contrast [26].

Hence, the reassignment is going to re-focus the energy spread by the smoothing [23].

However, the reassignment application in timefrequency representation provides to run counter to its poor time-frequency concentration.

In this case the smoothing kernel $\phi_{\mathrm{TF}}(u, \Omega)$ is the Wigner-Ville distribution of some unit energy analysis window $h(t)$, with $\phi_{\mathrm{TF}}(u, \Omega)=\mathrm{WV}(h ; u, \Omega)$.

The values of the new position of energy contributions $(\hat{t}(x ; t, \omega), \widehat{\omega}(x ; t, \omega))$ are given by the center of gravity of the signal energy located in a bounded domain centered on $(t, \omega)$ and measured by the Rihaczek distribution. These coordinates are defined by the smoothing kernel $\phi_{\mathrm{TF}}(u, \Omega)$ and computed by means of short-time Fourier transforms in the following way [16]:

$$
\begin{aligned}
& \hat{\mathrm{t}}(\mathrm{x} ; \mathrm{t}, \omega)=\mathrm{t}-\frac{\iint \mathrm{u} \cdot \mathrm{WV}(\mathrm{h} ; \mathrm{u}, \Omega) \mathrm{WV}(\mathrm{x} ; \mathrm{t}-\mathrm{u}, \mathrm{w}-\Omega) \mathrm{du} \frac{\mathrm{d} \Omega}{2 \pi}}{\iint \mathrm{WV}(\mathrm{h} ; \mathrm{u}, \Omega) \mathrm{WV}(\mathrm{x} ; \mathrm{t}-\mathrm{u}, \mathrm{w}-\Omega) \operatorname{du} \frac{\mathrm{d} \Omega}{2 \pi}} \\
& =\mathrm{t}-\mathcal{R}\left\{\frac{\iint \mathrm{u} \cdot \mathrm{Ri}^{*}(\mathrm{~h} ; \mathrm{u}, \Omega) \operatorname{Ri}(\mathrm{x} ; \mathrm{t}-\mathrm{u}, \mathrm{w}-\Omega) \mathrm{du} \frac{\mathrm{d} \Omega}{2 \pi}}{\iint \mathrm{Ri}^{*}(\mathrm{~h} ; \mathrm{u}, \Omega) \operatorname{Ri}(\mathrm{x} ; \mathrm{t}-\mathrm{u}, \mathrm{w}-\Omega) \mathrm{du} \frac{\mathrm{d} \Omega}{2 \pi}}\right\} \\
& =\mathrm{t}-\mathcal{R}\left\{\frac{\operatorname{STFT}_{\mathrm{Th}}(\mathrm{x} ; \mathrm{t}, \omega) \cdot \mathrm{STFT}^{*}{ }_{\mathrm{h}}(\mathrm{x} ; \mathrm{t}, \omega)}{\left|\mathrm{STFT}_{\mathrm{h}}(\mathrm{x} ; \mathrm{t}, \omega)\right|^{2}}\right\} \\
& \widehat{\omega}(x ; t, \omega)=w-\frac{\iint \Omega \cdot W v(h ; u, \Omega) W V(x ; t-u, w-\Omega) d u \frac{d \Omega}{2 \pi}}{\iint W v(h ; u, \Omega) W V(x ; t-u, w-\Omega) d u \frac{d \Omega}{2 \pi}} \\
& =\omega-\mathcal{R}\left\{\frac{\iint \Omega \cdot \mathrm{Ri}^{*}(\mathrm{~h} ; \mathrm{u}, \Omega) \operatorname{Ri}(\mathrm{x} ; \mathrm{t}-\mathrm{u}, \mathrm{w}-\Omega) \mathrm{du} \frac{\mathrm{d} \Omega}{2 \pi}}{\iint \mathrm{Ri}^{*}(\mathrm{~h} ; \mathrm{u}, \Omega) \operatorname{Ri}(\mathrm{x} ; \mathrm{t}-\mathrm{u}, \mathrm{w}-\Omega) \mathrm{du} \frac{\mathrm{d} \Omega}{2 \pi}}\right\} \\
& =\omega+\operatorname{Im}\left\{\frac{\operatorname{STFT}_{\operatorname{Th}}(\mathrm{x} ; \mathrm{t}, \omega) \cdot \operatorname{STFT}^{*}{ }_{\mathrm{h}}(\mathrm{x} ; \mathrm{t}, \omega)}{\left|\operatorname{STFT}_{\mathrm{h}}(\mathrm{x} ; \mathrm{t}, \omega)\right|^{2}}\right\}
\end{aligned}
$$

With $\operatorname{Ri}(x ; t, \omega)=x(t) \cdot X^{*}(\omega) e^{-j \omega t}$

where $\delta(\mathrm{t})$ is the Dirac impulse.

For more explication, you can see Appendix of [16]. The corresponding equation to the reassignment operators is writing in the following way:

$$
\begin{aligned}
& M S_{h}=\iint S_{h}(x ; t, w) \delta\left(t^{\prime}-\hat{t}(x ; t, w)\right) \cdot \delta\left(w^{\prime}-\right. \\
& \widehat{w}(x ; t, w)) d t \frac{d w}{2 \pi}
\end{aligned}
$$

According to [16] this reassigned version is also nonnegative, and retains all the properties of the spectrogram except the bilinearity, still lost for the benefit of perfectly localized chirps and impulses.

Furthermore, the time and frequency corrections expressions (5) and (8) lead to a reliable computation of the reassignment operators.

We adopted in this work the reassignment method in order to obtain a clear and easily interpreted spectrogram.

\section{Log-Gabor filters}


Gabor filters offer an excellent simultaneous localization of spatial and frequency information [20]. They have many useful and important properties, in particular the capacity to decompose an image into its underlying dominant spectro-temporal components [17].

The log-Gabor filters coefficients contain relevant and effective information. The robustness of the proposed feature is explained by the fact that log-Gabor filters consist in signal decomposition into spectro- temporal atoms, which are efficient to form an approximate representation.The log-Gabor function in the frequency domain can be described by the transfer function $G(r, \theta)$ with polar coordinates [20]:

$$
G(r, \theta)=G_{\text {radial }}(r) \cdot G_{\text {angular }}(r)
$$

Where $G_{\text {radial }}(r)=e^{-\log \left(r / f_{0}\right)^{2} / 2 \sigma_{r}^{2}}$, is the frequency response of the radial component and $G_{\text {angular }}(r)=$ $\exp \left(-\left(\theta / \theta_{0}\right)^{2} / 2 \sigma_{\theta}^{2}\right)$, represents the frequency response of the angular filter component.

We note that $(r, \theta)$ are the polar coordinates, $f_{0}$ represents the central filter frequency, $\theta_{0}$ is the orientation angle, $\sigma_{r}$ and $\sigma_{\theta}$ represent the scale bandwidth and angular bandwidth respectively.

The log-Gabor feature representation $|S(x, y)|_{m, n}$ of a magnitude spectrogram $s(x, y)$ was calculated as a convolution operation performed separately for the real and imaginary part of the log-Gabor filters:

$$
\begin{gathered}
\operatorname{Re}(\mathrm{S}(\mathrm{x}, \mathrm{y}))_{\mathrm{m}, \mathrm{n}}=\mathrm{s}(\mathrm{x}, \mathrm{y}) * \operatorname{Re}\left(\mathrm{G}\left(\mathrm{r}_{\mathrm{m}}, \theta_{\mathrm{n}}\right)\right) \\
\operatorname{Im}(\mathrm{S}(\mathrm{x}, \mathrm{y}))_{\mathrm{m}, \mathrm{n}}=\mathrm{s}(\mathrm{x}, \mathrm{y}) * \operatorname{Im}\left(\mathrm{G}\left(\mathrm{r}_{\mathrm{m}}, \theta_{\mathrm{n}}\right)\right)
\end{gathered}
$$

$(x, y)$ represent the time and frequency coordinates of a spectrogram, and $m=1, \ldots, N_{r}=2$ and $n=1, \ldots, N_{\theta}=$ 6 where $N_{r}$ devotes the scale number and $N_{\theta}$ the orientation number. This was followed by the magnitude calculation for the filter bank outputs:

$|S(x, y)|=\sqrt{\left(\operatorname{Re}(S(x, y))_{m, n}\right)^{2}+\operatorname{Im}(S(x, y))_{m, n}}$

\section{Averaging outputs of log-Gabor filters}

The averaged operation was calculated for each $12 \mathrm{log}$ Gabor filter appropriate for each reassigned spectrogram; the purpose being to obtain a single output array [20]:

$$
|\widehat{S}(x, y)|=\frac{1}{N_{r} N_{\theta}} \sum_{\substack{m=1 \\ n=1}}^{N_{r}, N_{\theta}}|S(x, y)|_{m, n}
$$

\section{E. Mutual information}

The information found commonly in two random variables is defined as the mutual information between two variables $\mathrm{X}$ and $\mathrm{Y}$, and it is given as [27]:

$$
\mathrm{I}(\mathrm{X} ; \mathrm{Y})=\sum_{\mathrm{x} \in \mathrm{X}} \sum_{\mathrm{y} \in \mathrm{Y}} \mathrm{p}(\mathrm{x}, \mathrm{y}) \log \frac{\mathrm{p}(\mathrm{x}, \mathrm{y})}{\mathrm{p}(\mathrm{x}) \mathrm{p}(\mathrm{y})}
$$

Where $p(x)=\operatorname{Pr}(X=x)$ is the marginal probability density function and $p(x)=\operatorname{Pr}(X=x)$, and $p(x, y)=$ $\operatorname{Pr}(X=x, Y=y)$ is the joint probability density function.

\section{F. SVM Classification}

For the classification, we used a Support Vector Machine, in a One-against-One configuration. The SVM's is a tool for creating practical algorithms for estimating multidimensional functions [28].

In the nonlinear case, the idea is to use a kernel function $K\left(x_{i}, x_{j}\right)$, where $K\left(x_{i}, x_{j}\right)$ satisfies the Mercer conditions [29]. Here, we used a Gaussian RBF kernel whose formula is:

$$
\mathrm{k}\left(\mathrm{x}_{\mathrm{i}}, \mathrm{x}_{\mathrm{j}}\right)=\exp \left[\frac{-\left\|\mathrm{x}_{\mathrm{i}}-\mathrm{x}_{\mathrm{j}}\right\|^{2}}{2 \sigma^{2}}\right]
$$

Where $\|$.$\| indicates the Euclidean norm in \Re^{d}$ [30].

We hence adopted one approach of multiclass classification: One-against-One. This approach consists in creating a binary classification of each possible combination of classes, and the result for $k$ classes is $k(k-1) / 2$. The classification is then carried out in accordance with the majority voting scheme [31].

\section{ENVIRONMENTAL SOUND CLASSIFICATION SYSTEM BASED ON REASSIGNMENT METHOD AND LOG-GABOR FILTERS}

\section{A. Database Description}

The corpus sound samples used in the sound recognition experiments derived from different sound libraries available [32-33]. In addition, using several sound collections is important and very necessary to create a representative, large, and enough diverse databases.

Among the sounds of the corpus we find: explosions, broken glass, door slamming, gunshot, etc. This database includes impulsive and harmonic sounds for example phone rings $(\mathrm{Pr})$ and children voices $(\mathrm{Cv})$. We used 10 classes of environmental sounds as shown in Table 1. All signals have a resolution of 16 bits and a sampling frequency of $44100 \mathrm{~Hz}$ that is characterized by a good temporal resolution and a wide frequency band, which are both necessary to cover harmonic as well as impulsive sounds. When building database, great care was dedicated to the selection of the signals. Indeed, when a rather general use of the classification system is needed, some kind of intraclass diversity in the signal properties should be existed in the database. Besides, the chosen sound classes are given in Table 1, and they are integrally of surveillance applications.

We indicate also that the number of items in each class is intentionally not equal, and sometimes very different.

Furthermore, an efficient and suitable surveillance system should be able to correctly recognize these sounds with a view to lower the false alarm rate. In addition, we 
remark in this database the presence of some classes sound very similar to human listeners such as explosions (Ep) are pretty similar to gunshots (Gs), hence, it is sometimes not obvious to discriminate between them. They are deliberately differentiated to test capacity of the system in separating very similar classes of sounds.

A type of sounds is required by the application, sounds are non-still, mainly of short durations, mainly impulsive audio signals, and presenting a big diversity intra-classes and a lot of similarity inter-classes. Most of the impulsive signals introduced into the base have duration of $1 \mathrm{~s}$, but some sounds possess much superior durations which can achieve 6s (for certain samples of explosions and the Human screams).

Table1. Classes of sounds and number of samples in the database used for performance evaluation

\begin{tabular}{|c|c|c|c|}
\hline Classes & Train & Test & Total \\
\hline Door slams (Ds) & 208 & 104 & 312 \\
Explosions (Ep) & 38 & 18 & 56 \\
Glass breaking (Gb) & 38 & 18 & 56 \\
Dog barks (Db) & 32 & 16 & 48 \\
Phone rings (Pr) & 32 & 16 & 48 \\
Children voices (Cv) & 54 & 26 & 80 \\
Gunshots (Gs) & 150 & 74 & 224 \\
Human screams (Hs) & 48 & 24 & 72 \\
Machines (Mc) & 38 & 18 & 56 \\
Cymbals (Cy) & 32 & 16 & 48 \\
\hline Total & 670 & 330 & 1000 \\
\hline
\end{tabular}

"Fig.4"depicts environmental sounds spectrograms and their reassigned representation. Each class contains sounds with very different temporal or spectral characteristics, levels, duration, and time alignment. For example door slams (Ds) present a wide frequency band but with a short duration. In addition, for the children voices $(\mathrm{Cv})$, we can distinguish the presence of the privilege frequencies. Concerning phone rings $(\operatorname{Pr})$, we notice that they present fundamental frequencies. Furthermore, we notice that there are some similarities between explosions (Ep) and gunshots (Gs) although they belong to different classes. The used system should be able to discriminate sounds from these classes. Moreover, some used sounds present important energy content in the highest frequencies, for example glass breaking. Concerning the reassigned spectrogram as shown in figure 4, we notice a significant improvement in localization of the reassigned spectrogram in timefrequency domain in comparison to the spectrogram obtained by Short Time Fourier Transform. We can deduce that the reassignment method provides almost ideal representation of the environmental sound. We remark that the reassigned spectrogram provides good concentration at lower frequencies, but poor concentration at higher frequencies. In other words, the reassigned spectrogram obtained by the Short Time Fourier transform (STFT) enhances the concentration of the components in comparison to the spectrogram, and it does not contain any cross terms as shown in Figure 4 [34]. In addition, for reassignment spectrogram, we use the smoothing hanning window of length 256 with 192point overlap. In order to obtain reassigned spectrogram it

is necessary to compute auxiliary spectra and timefrequency corrections using the reassignment method of Auger and Flandrin previously described.
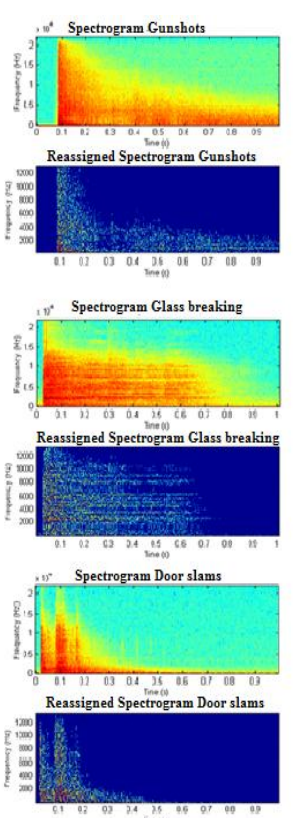

times
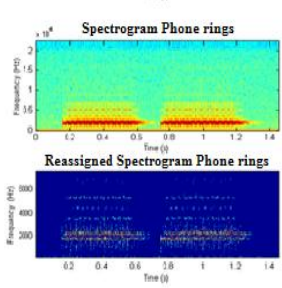

Fig.4. Spectrograms and their reassigned representation of 8 environmental sound classes

\section{B. SVM Classifier Design and Parameters Setting}

The SVM classifier design is previously described in Section 2. Thus, for multiclass classification, we evaluated the multiclass SVM (M-SVM) classifier Oneagainst-One learn to discriminate one class from another.

Furthermore, One-against-One classifiers are generally smaller and can be estimated using fewer resources than One-against-All classifiers. In the classification context with SVM many studies like [35], proved that Oneagainst-One classifiers are marginally more accurate than One-against-All classifiers. Hence, in this work we used One-against-One simply because is more suitable for practical use [31], and its training time is shorter than One-against-All. Most of the signals are impulsive.

We took $2 / 3$ for the training and $1 / 3$ for the test. Among the big problems met during the classification by the SVM's is the choice of the values of the kernel parameter $\gamma$ and the constant of regularization $C$. To resolve this problem we suggest the cross-validation procedure [36].

Indeed, according to [37], this method consists in setting up a grid-search for $\gamma$ and C. For the implementation of this grid, it is necessary to proceed iteratively, by creating a couple of values $\gamma$ and $\mathrm{C}$. 
The radial basis kernel was adopted for all the experiments. The parameter $\mathrm{C}$ was used also to determine the trade-off between margin maximization and training error minimization [38].

For the implementation of this grid, it is necessary to proceed iteratively, by creating a couple of values $\gamma$ and $C$. In this work, we use the following couples:

$C, \gamma: C=\left[2^{(-5)}, 2^{(-4)}, \ldots, 2^{(15)}\right]$ et $\gamma=\left[2^{(-15)}, 2^{(-14)}, \ldots, 2^{(3)}\right]$

The number of support vectors of our sound classes ranges between $(45, \ldots, 89)$.

\section{Experimental Results}

\section{- Results with Individual log-Gabor-filter}

The results of the first method are summarized in Table 2 , the classification rates for each single log-Gabor filter derived from reassigned spectrogram, which included 2 scales and 6 orientations, are relatively low, ranging from 51.78 to $99.35 \%$ for 10 sounds class.

The best classification result based on the first method belongs to the Door slams class with scale $=1$, and orientation=1 which is of the order $99.35 \%$.

In addition, we achieved an averaged recognition rate of order $81.94 \%$.

The reassignment method brings a good localization in time-frequency domain which ensures a high energy concentration. In order to improve the first method result, features should be extracted either from all log-Gabor filters or from a selected group of best performing filters [20].

Both the second and the third method are concentrated to improve and correct failures of first method.

\section{- Results with Concatenation of the 12 log-Gabor- filters}

In order to enlight the usefulness of the features presented in first method, we present classification results obtained when applying the concatenation of the $12 \mathrm{log}$ Gabor-filters to reassigned spectrogram in the feature vector.

Results of the second approach are illustrated in Table3. The obtained classification rate ranges from $85.71 \%$ to 95.83. We were able to achieve an averaged accuracy rate of the order $92.07 \%$ in ten classes with one-against-one approach.

Furthermore, this method leads to an increase approximately $10 \%$ of averaged recognition compared to the averaged result when we used single log Gabor filter method $(81.94 \%)$.

We can deduce that the concatenation of 12 log-Gabor filters contains and concentrates the useful information than single log-Gabor filter.

Table.2 Recognition Rates of 12 log-Gabor filters applied to one-against-one SVM's based classifier with Gaussian RBF kernel

\begin{tabular}{|c|c|c|c|c|c|c|c|c|c|c|c|}
\hline Scale & Orienta-tion & Ds & Ep & $\mathrm{Gb}$ & $\mathrm{Db}$ & $\operatorname{Pr}$ & $\mathrm{Cv}$ & Gs & $\mathrm{Hs}$ & $\mathrm{Mc}$ & $\mathrm{Cy}$ \\
\hline \multirow{6}{*}{1} & 1 & 99,35 & 62.50 & 57.14 & 83.33 & 68.75 & 78.57 & 89.28 & 92.64 & 80.35 & 93.75 \\
\hline & 2 & 96.15 & 55.35 & 60.71 & 79.16 & 70.83 & 82.14 & 89.73 & 89.70 & 82.14 & 89.58 \\
\hline & 3 & 95.83 & 62.50 & 66.07 & 83.33 & 72.91 & 83.92 & 93.30 & 95.58 & 89.28 & 93.75 \\
\hline & 4 & 99.03 & 55.35 & 67.85 & 81.25 & 81.25 & 78.57 & 88.39 & 92.64 & 78.57 & 85.41 \\
\hline & 5 & 98.39 & 62.50 & 51.78 & 79.16 & 75.00 & 83.92 & 86.16 & 86.76 & 76.78 & 83.33 \\
\hline & 6 & 99.03 & 58.92 & 51.78 & 81.25 & 79.16 & 81.25 & 85.71 & 86.76 & 80.35 & 83.33 \\
\hline \multirow{6}{*}{2} & 1 & 95.83 & 62.50 & 71.42 & 87.50 & 87.50 & 89.28 & 95.83 & 89.70 & 92.64 & 93.75 \\
\hline & 2 & 95.83 & 85.71 & 75.00 & 89.58 & 85.41 & 88.75 & 96.42 & 92.64 & 87.50 & 85.41 \\
\hline & 3 & 80.12 & 64.28 & 88.75 & 93.75 & 87.50 & 85.71 & 95.08 & 95.58 & 85.71 & 95.58 \\
\hline & 4 & 83.01 & 64.28 & 75.00 & 79.16 & 81.25 & 82.14 & 89.73 & 85.29 & 82.14 & 83.33 \\
\hline & 5 & 80.44 & 64.28 & 67.85 & 77.08 & 79.16 & 83.92 & 93.30 & 92.64 & 83.92 & 85.41 \\
\hline & 6 & 81.08 & 58.92 & 66.07 & 77.08 & 75.00 & 82.14 & 89.73 & 88.23 & 78.57 & 85.41 \\
\hline
\end{tabular}

Table.3 Recognition Rates for averaged outputs of Reassigned Spectrogram with 12 log-Gabor filters, 3 Reassigned Spectrogram patches applied to one-against-one SVM's based classifier with Gaussian RBF kernel

\begin{tabular}{|c|c|c|c|c|}
\hline \multirow[b]{2}{*}{ Classes } & \multicolumn{2}{|c|}{$\begin{array}{c}\text { Reassigned Spectrogram with } 12 \text { log-Gabor filters } \\
\text { concatenation }\end{array}$} & \multicolumn{2}{|c|}{3 Reassigned Spectrogram patches } \\
\hline & $\begin{array}{c}\text { Parameters Kernel } \\
(c, \gamma)\end{array}$ & Classif. Rate (\%) & $\begin{array}{l}\text { Parameters Kernel } \\
(c, \gamma)\end{array}$ & Classif. Rate (\%) \\
\hline Ds & $\left(2^{(-1)}, 2^{(-6)}\right)$ & 95.83 & $\left(2^{(-5)}, 2^{(-6)}\right)$ & 94.87 \\
\hline Ep & $\left(2^{(-1)}, 2^{(2)}\right)$ & 85.71 & $\left(2^{(-4)}, 2^{(-6)}\right)$ & 88.75 \\
\hline $\mathrm{Cb}$ & $\left(2^{(2)}, 2^{(3)}\right)$ & 91.07 & $\left(2^{(-5)}, 2^{(2)}\right)$ & 78.57 \\
\hline $\mathrm{Db}$ & $\left(2^{(-3)}, 2^{(-14)}\right)$ & 93.75 & $\left(2^{(1)}, 2^{(3)}\right)$ & 89.58 \\
\hline $\operatorname{Pr}$ & $\left(2^{(-3)}, 2^{(-7)}\right)$ & 93.75 & $\left(2^{(15)}, 2^{(1)}\right)$ & 93.75 \\
\hline $\mathrm{Cv}$ & $\left(2^{(-4)}, 2^{(2)}\right)$ & 88.75 & $\left(2^{(-1)}, 2^{(-6)}\right)$ & 85.71 \\
\hline Gs & $\left(2^{(10)}, 2^{(-5)}\right)$ & 93.30 & $\left(2^{(-4)}, 2^{(2)}\right)$ & 95.83 \\
\hline Hs & $\left(2^{(-1)}, 2^{(-6)}\right)$ & 95.58 & $\left(2^{(-3)}, 2^{(-4)}\right)$ & 95.58 \\
\hline $\mathrm{Mc}$ & $\left(2^{(10)}, 2^{(-1)}\right)$ & 89.28 & $\left(2^{(-4)}, 2^{(-6)}\right)$ & 92.85 \\
\hline $\mathrm{Cy}$ & $\left(2^{(-5)}, 2^{(2)}\right)$ & 93.75 & $\left(2^{(-3)}, 2^{(-7)}\right)$ & 93.30 \\
\hline
\end{tabular}




\section{- Results with 12 log-Gabor-filters applied to the three patch reassigned spectrogram}

Results of third approach are shown in Table 3. Besides, we obtained in this approach an averaged accuracy rate of the order $90.87 \%$. This result is better than the first method result but is slightly lower than the second method result. Also, this method leads to an increase approximately $11 \%$ of averaged recognition compared to the result obtained when we applied single log-Gabor filters.

\section{- Comments and Discussion}

The reassignment method depends on the proper choice of smoothing kernel in order to produce simultaneously a high concentration of the signal components [16]. It permits to build a readable timefrequency representation process.

Moreover, the use of reassignment approach ensures an easily interpretation of spectrogram signature.

Nevertheless, previous studies [23-24-25] show that using reassignment method can improve the detection, the additive sound modeling, the segmentation and the classification performance.

The reassignment method allows removing the signal interference refocuses and concentrates the useful information. In this case it ensures good time-frequency decomposition.

Moreover, the use of reassignment method and logGabor filters offer the best simultaneous localization of spatial and frequency information. Generally, log-Gabor filters don't have DC component, which helps to improve the contrast of the reassigned spectrogram. In addition, the transfer function of the Log-Gabor function has an extended tail at the high frequency end, which allows us to achieve wide spectral information with localized spatial extent [39].

Besides, SVMs have proven to be robust in high dimensions. They are well founded mathematically to reach good generalization while keeping high classification accuracy.

The performance of the proposed classification system has been evaluated with SVMs by using a set of synthetic test signals. However, proposed methods maintain overall good performance.

The experiments results are satisfactory, which encourages us to investigate better in the reassignment method.

\section{Comparaison of state-of-the-art methods}

In this section, our experimental result was compared to the state-of-the-art methods results. Table 5 illustrated different methods for environmental sounds recognition with their classification rate. We begin by the method tested in [40], which used acoustic features, the combination between 13 MFCCs, 1 RASTA-PLP, 5 Amplitude Descriptor (AD), 1 Spectral Flux (SF), 1 Loudness, has given an average classification rate of the order $88.2 \%$. Then, in the work of D. Istrate et al. [9], which used as features $16 \mathrm{MFCCs}+$ energy $+\Delta+\Delta \Delta$, the recognition rate is of the order $89.3 \%$.
Furthermore, the studies of Chu et al. [1] proposed an approach based on combination of matching pursuit (MP) and MFCCs features. This combination gives the average classification rate of $83.9 \%$ in discriminating fourteen classes with GMM classifier.

When comparing these previous methods results with our classification system, we remark that our system has significant and better results. The experimental results listed in Table 4 demonstrate our efficient features for environmental sound recognition. Our classification rate is of the order $92.07 \%$ when we used reassigned spectrogram with 12 log-Gabor filter concatenation.

To conclude, we compared also our obtained results with the results obtained by Rabaoui et al.[2], who used a combination between MFCCs, energy, Log energy, SRF (SpectralRoll-Off-Point and SC (Spectral Centroide). This method has given an average classification rate of $(90.64 \%)$

in the nine environmental sounds classes. With the same nine sounds classes (Door slams (Ds), Explosions (Ep), Glass breaking (Gb), Dog barks (Db), Phone rings (Pr), Children voices ( $\mathrm{Cv}$ ), Gunshots (Gs), Human screams (Hs), Machines (Mc)), and using 12 log-Gabor filters concatenation our recognition accuracy is of the order $(91.89 \%)$. Our result shows that our features are significantly better in spite of their limited number. This can be partly explained by the fact that log-Gabor filters and reassignment method have the advantage of the best simultaneous localization of spatial and frequency information.

Nevertheless, as shown in Table 4, the classical method used various audio features combination, with selection criteria but here we opened a new research area using reassigned spectrogram as image texture for everyday sounds classification.

As can be seen in Table 4, our adopted features lead to an important improvement of the recognition accuracy when compared with the result done by classical features. According to the experimental results, our methods achieved enhanced performances in the visual domain.

Table.4 Comparison of state-of-the-art methods

\begin{tabular}{|l|l|}
\hline Features & Classification Rate \\
\hline $\begin{array}{l}\text { 13 MFCCs, 1 RASTA-PLP, } \\
\text { Flux (SF), 1 Loudness [40] } \\
\text { Fmplitude Descriptor (AD), Spectral }\end{array}$ & $88.2 \%$ \\
\hline 16 MFCCs+energy+ $+\Delta \Delta[9]$ & $89.3 \%$ \\
\hline Matching Pursuit (MP) + MFCCs [1] & $83.14 \%$ \\
\hline $\begin{array}{l}\text { MFCCs+energy+Log } \\
\text { energy+SRF(SpectralRoll-Off- } \\
\text { Point+SC(Spectral Centroide) [2] }\end{array}$ & $90.64 \%$ \\
\hline $\begin{array}{l}\text { Adopted Descriptors using 12 log-Gabor } \\
\text { filters }\end{array}$ & $92.07 \%$ \\
\hline
\end{tabular}

The adjustment of the visual features extraction methods used in image processing to the special characteristics of the environmental sounds have given 
satisfactory and improved classification results. Using reassignmen method, log-Gabor filters, a supervised method of classification (SVM) and a mutual information [41] gives the best discrimination between specific sound classes $(92.07 \%)$.

\section{CONCLUSION}

In this paper, a robust approach for improving the energy concentration of signals in the time-frequency domain has been proposed and tested to three new methods for environmental sound classification. This approach is based on the idea of reassigning the timefrequency representation of signal in order to obtain sharper representation.

We focused on novel sets of relevant features based on reassigned spectrogram. All methods used spectrotemporal components derived from log-Gabor filters. The first method uses a single log-Gabor filter. The second method uses an average of 12 log-Gabor filters concatenation. The third method segmented reassigned spectrogram into three patches with average of 12 logGabor filters. The two last methods showed promising results in the process of surveillance applications in the environmental sounds.

Our obtained results proved that the reassignment method is very efficient and it can be applied to a wide class of time frequency distributions.

In addition, this paper deals with robust features used with one-against-one SVM-based classifier in order to have a system that quietly works, independent of recording conditions. In all methods, the average highest classification accuracy was achieved while using the reassigned spectrogram with 12 log-Gabor filters concatenation $(92.07 \%)$.

Future research directions will include other methods extracted from image processing to apply in environmental sound classification.

\section{REFERENCES}

[1] S. Chu, S. Narayanan, and C.C.J Kuo, "Environmental Sound Recognition with Time-Frequency Audio Features", IEEE Trans. on Speech, Audio, and Language Processing, Vol. 17, No.6, 2009, pp.1142-1158.

[2] A. Rabaoui, M. Davy, S. Rossignol, and N. Ellouze, "Using One-Class SVMs and Wavelets for Audio Surveillance", IEEE Transactions on Information Forensics And Security, Vol.3, No.4, 2008, pp.763-775.

[3] V. Peltonen, J. Tuomi, A. Klapuri, J. Huopaniemi, and T. Sorsa, "Computational audiroty scene recognition", Int. Conf.Acoustics, Speech Signal Processing, 2002, pp.1941-1944.

[4] M. Vacher, D. Istrate, L. Besacier, J. F. Serignat, and E. Castelli, "Sound detection and classification for medical telesurvey", In Proc. IASTED Biomedical Conf., Innsbruck, Autriche, 2004, pp.395-399.

[5] A. Dufaux, L. Besacier, M. Ansorge, and F. Pellandini, "Automatic Sound Detection and Recognition For Noisy Environment", In Proceedings of European Signal Processing Conference (EUSIPCO), 2000, pp.1033-1036.
[6] A. Fleury, N. Noury, M. Vacher, H. Glasson and J.F Serignat, "Sound and speech detection and classification in a Health Smart Home", 30th IEEE Engineering in Medicine and Biology Society (EMBS), 2008, pp. 46444647.

[7] D. Mitrovic, M. Zeppelzauer, H. Eidenberger, "Analysis of the Data Quality of Audio Descriptions of Environmental Sounds", Journal of Digital Information Management (JDIM), Vol.5, No.2, 2007, pp.48-54.

[8] K. El-Maleh, A. Samouelian, and P. Kabal, "Frame-level noise classification in mobile environments", In Proc. ICASSP,1999, pp.237-240.

[9] D. Istrate, "Détection et reconnaissance des sons pour la surveillance médicale", PhD thesis, INPG, France, 2003.

[10] G.Yu, and J.J. Slotine, "Fast Wavelet-based Visual Classification", In Proc. IEEE International Conference on Pattern Recognition. ICPR, 2008, pp.1-5.

[11] G.Yu, and J.J. Slotine, "Audio Classification from TimeFrequency Texture”, In Proc. IEEE. ICASSP, 2009, pp.1677-1680.

[12] J. Dennis, and H.D. Tran, and H. Li, "Spectrogram Image Feature for Sound Event Classification in Mismatched Conditions", Signal Processing Letters, IEEE, Vol.18, No.2, 2011, pp.130-133.

[13] T. Ezzat, J. Bouvrie and T. Poggio, "Spectro-Temporal Analysis of Speech Using 2-D Gabor Filters", Proc. Interspeech, 2007, pp.1-4.

[14] S. Souli, Z. Lachiri, "Environmental Sounds Classification Based on Visual Features", CIARP, Vol. 7042, Springer, Chile, 2011, pp.459-466.

[15] R. Kelly Fitz, A. Sean Fulop, "A unified theory of timefrequency reassignment", Computing Research Repository- CORR, abs/0903.3, 2009.

[16] F. Auger and P. Flandrin, "Improving the Readability of Time-Frequency and Time- Scale Representations by the Reassignment Method”, IEEE Trans. Signal Proc.,Vol.40, No.5,1995 pp.1068-1089.

[17] M. Kleinschmidt, "Methods for capturing spectrotemporal modulations in automatic speech recognition", Electrical and Electronic Engineering Acoustics, Speech and Signal Processing Papers, Acta Acustica, Vol.88, No.3, 2002, pp. 416-422.

[18] M. Kleinschmidt, "Localized spectro-temporal features for automatic speech recognition", In Proc. Eurospeech, 2003, pp.2573-2576.

[19] L. He, M. Lech, N. C. Maddage and N Allen, "Stress Detection Using Speech Spectrograms and Sigma-pi Neuron Units", Int. Conf. on Natural Computation, 2009, pp.260-264.

[20] L. He, M. Lech, N. Maddage, N. Allen, "Stress and Emotion Recognition Using Log-Gabor Filter", Affective Computing and Intelligent Interaction and Workshops, ACII, 3rd International Conference on, 2009, pp.1-6.

[21] Z. Xinyi, Y. Jianxiao, H. Qiang, "Research of STRAIGHT Spectrogram and Difference Subspace Algorithm for Speech Recognition", $2^{\text {nd }}$ Int. Congress On Image and Signal Processing (CISP), IEEE, 2009, pp.1-4.

[22] J. Dennis, and H.D. Tran, and H. Li, "Image Representation of the Subband Power Distribution for Robust Sound Classification", Proc. INTERSPEECH, ISCA, 2011, pp.2437-2440.

[23] F. Millioz, N. Martin, "Réallocation du spectrogramme pour la détection de frontières de motifs temps-fréquence", Colloque GRETSI, 2007, pp.11-14.

[24] K. Fitz and L. Haken, "On the Use of Time-Frequency Reassignment in Additive Sound Modeling”, J.Audio Eng. 
Soc (AES), Vol.50, No.1, 2002, pp.879-893.

[25] F. Millioz, N. Martin, "Reassignment Vector Field for Time-Frequency Segmentation", $14^{\text {th }}$ international congress on sound and vibration, ICSV 14, 2007.

[26] E. Chassande-Mottin, "Méthodes de réallocation dans le plan temps-fréquence pour l'analyse et le traitement de signaux non stationnaires", PhDhesis, CergyPontoiseUniversity, 1998.

[27] N. Kwak, C. Choi, "Input Feature Selection for Classification Problems", IEEE Trans, On Neural Networks, Vol.13, No.1, 2002, pp.143-159.

[28] V. Vladimir, and N. Vapnik, "An Overview of Statistical Learning Theory", IEEE Transactions on Neural Networks, Vol.10, No.5, 1999, pp.988-999.

[29] V. Vapnik, and O. Chapelle, "Bounds on Error Expectation for Support Vector Machines", Journal Neural Computation, MIT Press Cambridge, MA, USA, Vol.12, No.9, 2000, pp.2013-2036.

[30] B. Scholkopf, and A. Smola, "Learning with Kernels", MIT Press, 2001.

[31] C.-W Hsu, C.-J Lin, "A comparison of methods for multiclass support vector machines", J. IEEE Transactions on Neural Networks, Vol.13, No.2, 2002, pp.415-425.

[32] Leonardo Software website. [Online]. Available: http ://www.leonardosoft.com. Santa Monica, CA 90401.

[33] Real World Computing Paternship, Cd-sound scene database in real acoustical environments, 2000, http://tosa.mri.co.jp/ sounddb/indexe.htm.

[34] E. Sejdi, I. Djurovi, and J. Jiang, "Time--frequency feature representation using energy concentration: An overview of recent advances", Digit. Signal Process. Vol.19, No. 1, 2009, pp. 153-183.

[35] J. Weston and C. Watkins, "Support vector machines for multi-class pattern recognition", 7th Eur Symp. Artificial Neural Networks, Vol.4, No.6, 1999, pp. 219-224.

[36] I. L Kuncheva, "Combining Pattern Classifiers Methods and Algorithms", ISBN 0-471-21078-1 (cloth). A WileyInterscience publication, Printed in the United States of America. TK7882.P3K83, 2004.

[37] C.-W. Hsu, C-C. Chang, C-J Lin, "A practical Guide to Support Vector Classification, Department of Computer Science and Information Engineering National Taiwan University", Taipei, Taiwan. Available: www.csie.ntu.edu.tw/ cjlin/, 2009.

[38] J-C. Wang, H-P Lee, J-F Wang, and C-B Lin, "Robust Environmental Sound Recognition for Home Automation", Automation Science and Engineering, IEEE Transactions on, Vol.5, No.1, 2008, pp. 25-31.

[39] S. Mehdi Lajevardi, Z. M. Hussain, "Facial Expression Recognition Using Log-Gabor Filters and Local Binary Pattern Operators", International Conference On Communication, Computer and Power (ICCCP'09), Muscat February15-18, 2009.

[40] D. Mitrovic, M. Zeppelzauer, H. Eidenberger, "Towards an Optimal Feature Set for Environmental Sound Recognition”, Technical Report TR-188-2, 2006.

[41] G. Kattmah,G. A. Azim, "Identification Based on Mutual Information and Neural Networks", International Journal on Image Graphics and Signal Processing, Vol.9, pp.5057, 2013.

Souli S. Sameh Birth in Tunis, on 26/12/1980. Assistant at Higher Institute of Arts and Multimedia Manouba (ISAMM), Tunis. Have a thesis degree in image and signal processing on
14 March 2014 at national school of Engineers of Tunis (ENIT), Tunisia.

She has a master in Electronics: Automatic and signal processing, National School of Engineers of Tunis (ENIT), Tunis, Tunisia in October 2008. She has a master's degree in Computer Science, Faculty of Sciences of Tunis (FST), Computer Science Department, Tunisia in June 2005. She is Assistant for six years at Higher Institute of Arts and Multimedia Manouba (ISAMM), she has a journal paper named: "Multiclass support vector machines for environmental sounds classification in visual domain based on log-Gabor filters" at Int J Speech Technol, 2013. She has a book chapter named: "Environmental Sounds Classification Based on Visual Features" at CIARP, 2011. Dr. Souli is a review in Science Publishing Group journal. 